\title{
Neuroprotective Effect of the Recombinant Human Erythropoietin in the Entorhinal Cortex and Thalamus of Rats Exposed to Focal Cerebral Ischemia
}

\author{
Jasenka Mrsic Pelcic ${ }^{1, *}$, Maja Rukavina ${ }^{1}$, Goran Pelcic ${ }^{2}$, Vitezić Dinko \\ Kristina Pilipovic ${ }^{1}$
}

\begin{abstract}
Objective: Neuroprotective potential of recombinant human erythropoietin (rhEpo) was reported in various experimental models of brain damage but the exact mechanism of its effect is still unclear. In the present study, the effect of rhEpo administration on the level of neuronal loss and neurodegenerative changes in the dorsolateral band of the entorhinal cortex and ventral posteriomedial nucleus of the thalamus in rats following focal cerebral ischemia was examined.

Methods: Focal cerebral ischemia was induced in male Hanover Wistar rats (250$350 \mathrm{~g}$ ) by right middle cerebral artery occlusion (MCAO) model for $1 \mathrm{~h}$. After 23 $\mathrm{h}$ of reperfusion, ischemic animals were sacrificed and the neuronal damage was detected using the Fluoro Jade B fluorescent staining to detect neurodegeneration, together with NeuN immunostaining used to detect neuronal loss. Ischemic animals received either vehicle or rhEpo (5000 IU/kg, intraperitoneally) $3 \mathrm{hrs}$ after MCAO, and were sacrificed $21 \mathrm{~h}$ later. Sham operated; vehicle treated animals served as the control group.
\end{abstract}

Results: Administration of rhEpo significantly increased the NeuN immunoreactivity in the entorhinal cortex compared to the neuronal loss detected in ischemic, non-treated animals and decreased the number of Fluoro Jade B positive neurons in comparison to neuronal damage detected in ischemic, nontreated animals. The effect of rhEpo treatment in thalamus was not significant.

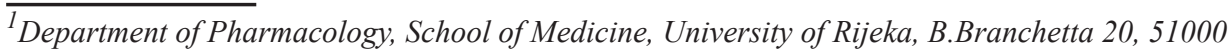
Rijeka, Croatia

${ }^{2}$ Clinical Hospital Centre Rijeka, Clinics for Ophthalmology, Krešimirova 42, 51000 Rijeka, Croatia

* Corresponding author:

Mršić-Pelčić Jasenka jasenka.mrsic.pelcic@uniri.hr ORCID: 0000-0002-6313-9232

* Received: 25 April 2021 Accepted: 16 June 2021

DOI: 10.17932/EJOH.2020.022/ejoh_v02i1002
} 
Neuroprotective Effect of the Recombinant Human Erythropoietin in the Entorhinal Cortex and Thalamus of Rats Exposed to Focal Cerebral Ischemia

Conclusion: Our results indicated that focal cerebral ischemia caused neuronal damage in the entorhinal cortex and that rhEpo treatment was effective in preventing above mentioned alterations.

Keywords: recombinant human erythropoietin (rhEpo), focal cerebral ischemia, neuroprotection, neuronal loss, neurodegeneration

\section{Introduction}

Acute ischemic stroke is one of the major causes of death and disabilities worldwide. The majority of ischemic human strokes are caused by middle cerebral artery occlusion (MCAO) (1). The MCAO model, experimentally induced in rodents by the intraluminal suture method, is well-standardized animal model of focal cerebral ischemia. This model effectively mimic clinical changes that occur during and after human ischemic stroke (2). In addition, it is considered as a reliable model to test a complex pathophysiology of cerebral ischemia such as a potential neuroprotective effect of various drugs.

It is well known that during focal cerebral ischemia neurons and glial cells are severely damaged by compelx cascade of pathophysiological events starting with periinfarct depolarization and overproduction of reactive oxygen species (ROS). Consequent oxidative stress followed by more delayed postischemic inflammation cause mitochondrial damage, activation of apoptotic proteins, cytochrome c release, and caspase activation. These events finally result in apoptotic cell death within several days/weeks after cerebral ischemia and are involved in the progression and expansion of brain injury $(3,4)$.

Thus, the administration of drugs that have potential to control one or more elements of the above mentioned pathophysiological cascade during focal cerebral ischemia is important for achieving neuroprotection against ischemia/ reperfusion injury.

Erythropoietin (Epo) is hematopoietic growth factor mostly secreted by the kidneys in response to tissue hypoxia (5). Its crucial role in the regulation of process of erythropoiesis stimulation is well known, but a significant interest in its effect came after it was found that Epo and erythropoietin receptors (EpoR) are expressed locally in response to the brain ischemia. It is assumed that they act as potential endogenous neuroprotectants excreted in response to oxidative stress, especially in the penumbra. This pointed out possibility that exogenous administration of rhEpo could contribute to endogenous neuroprotective response of ischemic brain tissue $(6,7)$. Effective clinical application for the treatment of anemia in renal failure and cancer was well described $(8,9)$ but it was shown 
also that rhEpo acts as multifunctional drug that could potentiate endogenous neuroprotective effects as a consequence of activation of various biochemical pathways. This provide anti-apoptotic, anti-oxidant and anti-inflammatory effect as well as the stimulation of angiogenic and neurogenic mechanisms (10). The capability of rhEpo to cross the blood-brain barrier after systemic administration, its acceptable therapeutic window and good clinical tolerability are additional advantages for its potential application in human stroke therapy $(11,12)$. However, the exact mechanisms of its neuroprotection are still not completely understood and outcomes from conducted clinical trials are controversial $(13,14)$.

In this study, in order to evaluate the potential neuroprotective effect of rhEpo against ischemia/reperfusion injury, we examined the effects of a) focal cerebral ischemia/reperfusion on the potential neuronal loss and neurodegenerative changes in the entorhinal cortex and thalamus of rats exposed to transient middle cerebral artery occlusion and b) systemic administration of $5000 \mathrm{IU} / \mathrm{kg}$ of $\mathrm{rhEpo}$ on above mentioned parameters.

\section{Materials and Methods}

\section{Experimental animals}

Male Hannover-Wistar rats, weighing 250-350 g were used in this study. Rats were maintained on a $12 \mathrm{~h}$ light-dark cycle and allowed free access to food and water. All experiments were performed between 9 a.m. and 3 p.m. in a silent room, at a temperature of $22-24^{\circ} \mathrm{C}$. All experimental procedures involving animals were approved by the Faculty's Ethical Committee and were carried out in accordance with the Croatian laws and rules (NN135/06; NN 37/13; 55/13) and with the guidelines set by the European Community Council Directive (2010/63/EU). All efforts were made to minimize animals' suffering and to reduce the number of animals used in experiments. This research was supported by University of Rijeka under project uniri-biomed-18-115 1253.

\section{Experimental design}

The rats were randomly divided into three experimental groups. The first group was sham control, vehicle-treated $(n=4)$, the second group was MCAO, vehicletreated $(\mathrm{n}=3)$, and the third group was rhEPO (Eprex $2000 \mathrm{IU} / 0,5 \mathrm{ml}$, Janssen Biologics B.V., Leiden, Netherlands) treated $(n=4)$. The vehicle or rhEPO were administered at $3 \mathrm{~h}$ following ischemia induction, rats were sacrificed, and their brains were isolated $24 \mathrm{~h}$ post-MCAO induction.

Surgical procedure for the transient middle cerebral artery occlusion

Rats were anesthetized with $350 \mathrm{mg} / \mathrm{kg}$ of chloral hydrate administered intraperitoneally (i.p.). The cerebral ischemia-reperfusion injury was performed 
by the right MCAO for $1 \mathrm{~h}$. MCAO was performed by intraluminal nylon suture occlusion method as described by Longa et al. (15). and with modifications suggested by Belayev et al. (16). Briefly, under an operating microscope, the right common carotid artery was exposed and carefully dissected from the surrounding nerves and fascia. The internal carotid artery was isolated and separated from the adjacent vagal nerve, and the pterygopalatine artery was ligated close to its origin with a 5-0 nylon suture. Next, a 4-0 silk suture was tied loosely around the mobilized external carotid artery stump, and a 3-0 monofilament nylon suture of approximately $4 \mathrm{~cm}$ length, prepared by blunting the tip of the suture by heating it near a flame, was inserted through the proximal external carotid artery into the internal carotid artery and subsequently into the circle of Willis, effectively occluding the middle cerebral artery (MCA). The suture was inserted about 18-20 $\mathrm{mm}$ from the bifurcation of the common carotid artery after which the neck incision was closed. The body temperature was maintained at $37 \pm 0.5^{\circ} \mathrm{C}$ with a heating pad and rectal probe. After $1 \mathrm{~h}$ of MCAO, the intraluminal suture was carefully removed, and the internal carotid artery was reperfused. Shamoperated, vehicle-treated rats served as the control groups. In these animals, the internal carotid artery was only isolated, but the MCA was not occluded. Once awakened from the anesthesia, the animals were returned to their home cages. Animals of all experimental groups were sacrificed $24 \mathrm{~h}$ after the induction of $\mathrm{MCAO}$ or sham experimental procedure.

\section{Histological and immunohistochemical analyses}

Histological and immunohistochemical analyses were performed in the dorsolateral band of the entorhinal cortex and ventral posteriomedial nucleus of the thalamus in rats of control, vehicle treated as well as ischemic animals that were injected with either vehicle or rhEpo. For these analyses, animals were perfused transcardially with $4 \%$ paraformaldehyde in phosphate-buffered saline. Brains were removed and stored in the fixative solution for $20 \mathrm{~h}$ at $4^{\circ} \mathrm{C}$ and subsequently embedded in paraffin.

For the neuronal loss determination, immunohistochemical detection of NeuN stained cells was performed on $3 \mu \mathrm{m}$ paraffin sections. Following deparaffinization and rehydration of the sections, antigen retrieval was achieved by microwaving slides in citric acid buffer $(10 \mathrm{mM}, \mathrm{pH} 6.0)$. Following the blocking step with $5 \%$ normal rabbit serum and $1 \%$ bovine serum albumin in TBSTriton X-100 (0.025\%), sections were incubated with mouse monoclonal antiNeuN primary antibody (Millipore, Billerica, MA, USA), overnight at $4^{\circ} \mathrm{C}$. The next day biotinylated secondary rabbit anti-mouse antibody (Dako Cytomation, Glostrup, Denmark) was applied for $2 \mathrm{~h}$, followed by $30 \mathrm{~min}$ incubation with red fluorescence emitting DyLight 594 ${ }^{\mathrm{TM}}$-conjugated streptavidin (Vector Laboratories, Burlingame, CA, USA). 
Microphotographs of the designated brain regions were obtained using the Olympus BX 51microscope equipped with an Olympus DP 70 digital camera (Olympus, Japan).

Fluoro-Jade B fluorescent staining was used to study neurodegenerative changes in the investigated brain regions. Brain slices were first deparaffinized in xylene and then rehydrated in decreasing concentrations of ethanol to deionized water $(\mathrm{dH} 2 \mathrm{O})$. Slides were then subjected to $0.06 \%$ potassium permanganate solution for 10 minutes, rinsed twice with $\mathrm{dH} 2 \mathrm{O}$ for 1 minute, and incubated in $0.001 \%$ Fluoro-Jade B (Millipore, Billerica, MA) solution diluted in $0.1 \%$ acetic acid, for 20 minutes in the dark. Slides were washed 3 times and air-dried at room temperature (RT) overnight. The next day, after clearing in xylene, the slides were mounted in Entellan (Merck Millipore) and coverslipped.

\section{Photo acquisition and image analysis}

In the histological analyses, all slides were examined by epifluorescence microscopy using the appropriate light filter cubes (Olympus BX51 microscope and Olympus DP 71 CCD digital camera; Tokyo, Japan). Microphotographs of the coronal sections of the rat brain were taken at approximately -3.12 to -3.60 $\mathrm{mm}$ relative to bregma, according to Paxinos and Watson (2005) (17). All the image processing and analyses were performed manually by a blind investigator by using the ImageJ software (NIH, Bethesda, MD, USA). For the thalamus, photographs were taken within the ventral posteromedial thalamic nucleus, and for the entorhinal cortex, the dorsolateral band was visualized.

For the quantification of the number of NeuN-immuno stained nuclei and the intensity of the Fluoro-Jade B staining, brain sections were photographed at $\times 400$ magnification. NeuN positive cells were counted within the visual field that was $0.143 \mathrm{~mm} 2$ in size. Quantification of the Fluoro-Jade B staining intensity was performed by calculating the densitometric mean of fluorescence intensity. Within each image, background fluorescent intensity was subtracted and mean gray intensity determined which resulted in the measurement of degenerating neurons within that field.

\section{Statistical analyses}

All the statistical analyses were performed using Statistica software version 13.0 (StatSoft Inc., Tulsa, OK, USA). Statistical significance for the number of NeuN positive cells, and the Fluoro Jade B intensity measurement were calculated according to the one-way analysis of variance, followed by Duncan's multiple range post-hoc test. All the results are expressed as means \pm standard error of means (SEM). In all the comparisons $\mathrm{p}<0.05$ was considered to indicate statistical significance. 
Neuroprotective Effect of the Recombinant Human Erythropoietin in the Entorhinal Cortex and Thalamus of Rats Exposed to Focal Cerebral Ischemia

\section{Results}

We examined the effects of focal cerebral ischemia/reperfusion as well as the influence of i.p. administration of $5000 \mathrm{IU} / \mathrm{kg}$ rhEpo injected $3 \mathrm{~h}$ after ischemic procedure on the neuronal loss and neurodegenerative changes in entorhinal cortex and thalamus of rats exposed to transient middle cerebral artery occlusion.

\section{Neuronal loss}

Figure 1 shows the NeuN immunoreactivity and the results of the semiquantitative analysis of number of NeuN positive cells in the dorsolateral band of the entorhinal cortex and ventral posteriomedial nucleus of the thalamus in rats of the control group and ischemic rats treated with either vehicle or rhEpo at $3 \mathrm{~h}$ after the induction of ischemia. Representative microphotographs show decreased NeuN immunoreactivity in the examined region of entorhinal cortex of the ischemic, vehicle treated animal compared to the rat of the control group. It is also apparent that the rhEpo treatment caused an increase in the NeuN immunostaining in the enthorinal cortex of the MCAO exposed animals (Figure 1a).. An overall ANOVA reveal a statistically significant difference among tested groups in enthorinal cortex $[F(2,8)=26,447 ; p<0,05]$ (Figure 1b).. Namely, the number of NeuN labeled cells in the enthorinal cortex was decreased in MCAO/Veh animals in comparison to the control group, $(443.68 \pm 57.75$ cells $/ \mathrm{mm} 2$ in the MCAO/Veh animals versus $599.86 \pm 36.42$ cells $/ \mathrm{mm} 2$ in the control animals). In the ischemic animals treated with rhEpo, the number of NeuN positive cells was 513.51 \pm 24.97 cells $/ \mathrm{mm} 2$ and it showed significant difference in comparison to the control or $\mathrm{MCAO} /$ Veh animals (Figure 1b). In our experimental conditions, an overall ANOVA did not reveal a statistically significant effect of the treatments on the number of NeuN immunopositive cells in thalamus [F $(2,8)=2,173 ; p>0,05]$ (Figure 1c and 1d)..

\section{Neurodegeneration}

The presence and extent of neurodegenerative changes were analyzed throughout the dorsolateral band of the entorhinal cortex and the ventral posteromedial nucleus of the thalamus using the Fluoro-Jade B histofluorescence. Within the both examined brain regions of the ischemic, vehicle-treated animals, FluoroJade $\mathrm{B}$ positive staining was detected in neuronal cell bodies and neuronal fibers (Figure 2a), contrary to the sections from the animal of the control group in which no degenerative changes were detected. From the microphotographs of the brain sections of the rhEpo injected ischemic rats, it is apparent that the investigated drug caused a slight decrease in the intensity of the Fluoro Jade B staining in both examined regions, but particularly in the dorsolateral entorhinal cortex. The quantification of the Fluoro-Jade B staining intensity in the dorsolateral entorhinal cortex revealed significant neurodegeneration in the animals exposed to MCAO 
A

Control

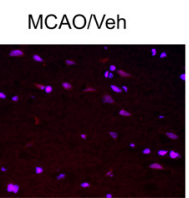

MCAONeh

C
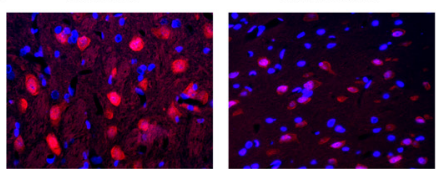

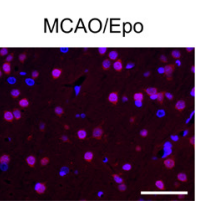

$\mathrm{MCAO/Epo}$

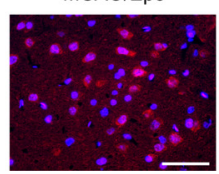

B

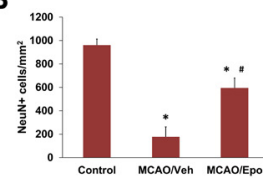

D

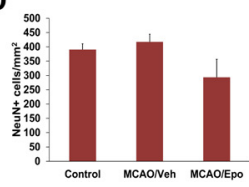

Figure 1. The effect of recombinant human erythropoietin treatment on the neuronal loss measurement in the dorsolateral band of the entorhinal cortex $(A, B)$ and ventral posteriomedial nucleus of the thalamus $(C, D)$ of rats exposed to transient cerebral ischemia. Representative microphotographs of the investigated regions of the entorhinal cortex (A) and the thalamus (C), immunofluorescently labeled with NeuN neuronal marker (red) from the rats sacrificed at $24 \mathrm{~h}$ after the induction of $1 \mathrm{~h}$ middle cerebral artery occlusion (MCAO) or sham operation. DAPI fluorescent dye (blue) was used as the nuclear counterstain. Sham-operated, vehicle-treated animals (Control) and the ischemic rats, treated with either vehicle (MCAO/Veh) or rhEpo (MCAO/Epo), were intraperitoneally injected at $3 \mathrm{~h}$ after the onset of the ischemic procedure or the end of the sham operation. Scale bar: $200 \mu \mathrm{m}$. Quantification of neuronal loss in the dorsolateral band of the entorhinal cortex (B) and ventral posteromedial nucleus of the thalamus (D) was done by counting the NeuN positive cells. Each value represents the mean \pm S.E.M. $(N=3-4) . * P<0.05$, significantly different from the Control group; ${ }^{*} P<0.05$, significantly different from the $M C A O /$ Veh group.

A

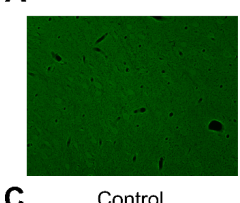

C

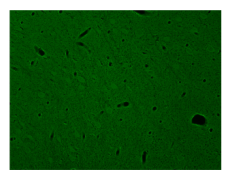

MCAO/Neh

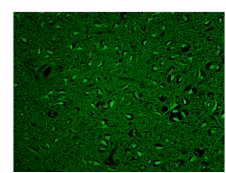

MCAO/Veh

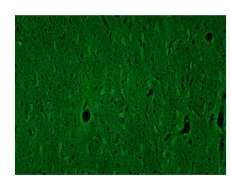

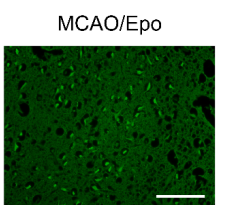

$\mathrm{MCAO/Epo}$

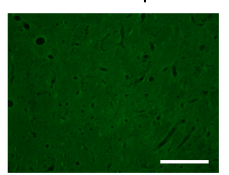

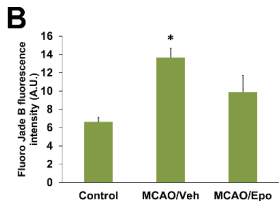

D

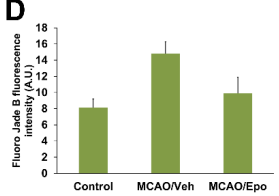

Figure 2. The effect of recombinant human erythropoietin treatment on the levels of neurodegenerative changes in the dorsolateral band of the entorhinal cortex $(A, B)$ and ventral posteriomedial nucleus of the thalamus $(C, D)$ of rats exposed to transient cerebral ischemia. Representative microphotographs of the investigated regions of the entorhinal cortex (A) and the thalamus $(C)$, from the brain sections stained with Fluoro Jade B fluorescent dye. Rats were sacrificed at $24 \mathrm{~h}$ after the induction of $1 \mathrm{~h}$ middle cerebral artery occlusion (MCAO) or sham operation. Sham-operated, vehicle-treated animals (Control) and the ischemic rats, treated with either vehicle (MCAO/Veh) or rhEpo (MCAO/Epo), were intraperitoneally injected at $3 \mathrm{~h}$ after the onset of the ischemic procedure or the end of the sham operation. Scale bar: $200 \mu \mathrm{m}$. Fluoro Jade $B$ staining in the dorsolateral band of the entorhinal cortex (B) and ventral posteromedial nucleus of the thalamus (D) was quantified as a measure of their fluorescence intensity and presented as arbitrary units (A.U.). Each value represents the mean \pm S.E.M. $(N=3-4) .{ }^{*} P<0.05$, significantly different from the Control group. 
$(\mathrm{p}<0.05)$ (Figure $2 \mathrm{~b})$ in comparison to the control animals. Also, rhEpo treatment caused a decrease in the Fluoro- Jade B staining intensity in this region but it was not significantly different from values determined in either control or $\mathrm{MCAO} / \mathrm{Veh}$ animals $[\mathrm{F}(2,8)=6,6075 ; \mathrm{p}<0.05]$. In the examined thalamic region, results of the quantitative analyses were evaluated statistically but the overall ANOVA did not reveal significant differences in the levels of Fluoro Jade B staining intensities between the investigated experimental groups $[F(2,8)=4,1727 ; p<0,05]$ (Figure $2 \mathrm{c}$ and $2 \mathrm{~d})$.

\section{Discussion}

In our study, we have examined extent of neuronal damage i.e., neuronal loss and/ or neurodegeneration in the dorsolateral band of the entorhinal cortex and ventral posteriomedial nucleus of the thalamus $24 \mathrm{~h}$ after induction of $1 \mathrm{~h} \mathrm{MCAO}$. In addition, the effect of rhEpo administration $3 \mathrm{~h}$ after induction of ischemia on the above-mentioned parameters was measured.

The results of presented experiments showed that focal cerebral ischemia induced by MCAO caused a statistically significant neuronal loss in dorsolateral cortex of experimental animals. Namely, registered decrease in NeuN staining in dorsolateral cortex of $\mathrm{MCAO} /$ vehicle treated animals was statistically significant in comparison to control animals. Our findings are in accordance with different published results. Thus, it was shown that MCAO followed by $24 \mathrm{~h}$ of reperfusion causes significant neuronal damage in various brain regions. The predominant affected areas are mostly striatum and cortex due to the lack of collateral blood supply $(2,18,19)$. It was described also that immunoreactivity for NeuN decrease significantly following MCAO (20), causing massive corticostriatal lesion with the peak of the damage $12-24 \mathrm{~h}$ following reperfusion $(20,21)$. Neuronal loss in our experimental model is in good correlation with statistically significant increase in neurodegeneration that we have found in experimental animals exposed to MCAO. Fluoro-Jade B positive neuronal cells in dorsolateral cortex revealed significant degeneration in ischemic animals exposed to MCAO in comparison to control animals according to Liu et al. (20). Duckworth et al. (22) have shown that a time course of escalating cortical neuronal degeneration was evident from 10 min to 7 days with a peak after 4 days following MCAO. On the contrary, Onken et al. (23) published results that have shown no Fluoro Jade staining $24 \mathrm{~h}$ after ischemic injury. They detected degenerative neurons 48-72 $\mathrm{h}$ post ischemia in different brain regions like hippocampus, striatum, and cortex. Similar neuronal damage in entorhinal cortex was described even up to 6 months following MCAO (24). Differences in time related neuronal damage following focal cerebral ischemia described above are mostly caused by different brain region sensitivity and suture placement technique. In conclusion, 
in our experimental model significant neuronal damage i.e., neuronal loss and neurodegeneration of the dorsolateral cortical neurons are described within first $24 \mathrm{~h}$ after $1 \mathrm{~h}$ MCAO.

In contrast to above mentioned findings, in our experimental model we did not find a significant neuronal damage, manifested neither by neuronal loss nor extent of neurodegeneration in the ventral posteriomedial nucleus of the thalamus of rats exposed to MCAO in comparison to control animals. The thalamus is a deep brain structure important for maintenance of normal neurological functions and transmission of various signals (25). In most animal models including MCAO, the thalamus is spared from the acute ischemic damage. Namely, the thalamus is primarily supplied by branches of the posterior cerebral arteries with minor contribution from the internal carotid artery. So, after MCAO, the brain damage is predominantly registered in regions supplied by the middle cerebral artery like cortex, striatum or globus pallidus. But it was shown also that brain damage following MCAO could occur even in deep regions such as thalamus because of the occlusion of deep and small cerebral arteries arising directly from the internal carotid artery $(25,26)$, proximally to the origin of middle cerebral artery. Consequently, this region could still be affected due to synaptic connections with the primary injury site. It was described that the widespread corticostriatal damage could result in retrograde and anterograde degeneration, from the perilesional cortex via the descending axons to the thalamus (27). This is probable explanation for results published by various authors demonstrating delayed neuronal damage even in the thalamus following $\operatorname{MCAO}(25,28)$. It was described that MCAO induced neuronal damage in regions within middle cerebral artery zone of irrigation, causes widespread edema that leads to secondary, delayed neuronal damage in the thalamus beginning 24 hours after induction of ischemia $(27,29,30)$. This delayed response of thalamic neuronal tissue is probable explanation for lack of significant neuronal damage in thalamus region in our experimental model.

Therapeutic strategies against cerebral ischemia are still very limited. The only clinically approved drug against acute ischemic stroke is tissue plasminogen activator (tPA) (31). However, tPA's narrow therapeutic time window (up to 4.5 hours after the induction of stroke) significantly reduces its' therapeutic potential in the treatment against focal cerebral ischemia (5). Various potential neuroprotective approaches have failed to reduce neuronal damage, neurological deficits, and mortality after cerebral ischemia, which leads to conclusion that discovery of novel therapies against focal cerebral ischemia are of the highest importance. It was shown that one of the promising therapeutic approaches in preventing neuronal damage might be administration of rhEpo $(3,6,7)$. According to Brines \& Cerami, several authors have confirmed that a different doses 
(500-5000 IU $/ \mathrm{kg}$ ) of rhEPO, administered systemically in rodents or primates, were reaching neuroprotective concentrations within various brain regions approximately $1 \mathrm{~h}$ after drug application (32). However, most of the experimental research in various animal models that examined the neuroprotective effects of different doses of rhEpo on brain damage, proposed $5000 \mathrm{IU} / \mathrm{kg}$ of rhEpo as optimal dosage (32). It was also shown that rhEpo applied at various time-periods ranging from pre-treatment to up to $24 \mathrm{~h}$ after ischemia, exerts the best results when applied within the first $3 \mathrm{~h}$ after induction of ischemia (18).

Administration of $5000 \mathrm{IU} / \mathrm{kg}$ of rhEpo in our experimental conditions, $3 \mathrm{~h}$ after induction of $1 \mathrm{~h} \mathrm{MCAO}$, significantly preserved the neuronal loss in dorsolateral cortex of ischemic animals in comparison to ischemic, vehicletreated rats. Namely, the number of NeuN staining neurons in ischemic animals treated with rhEpo is significantly higher in comparison to ischemic, vehicle treated animals. Administration of the drug was also effective in decreasing the number of cortical neurons undergoing neurodegenerative changes of ischemic animals although this result did not reach statistical significance in comparison to ischemic, vehicle treated animals. Administration or rhEpo did not reveal any significant changes in neuronal damage manifested as neuronal loss and/or neurodegeneration in thalamus of ischemic rats.

Neuroprotective effect of rhEpo application was revealed also by other authors although in different experimental conditions and in various brain regions. Various authors have shown that application of $5000 \mathrm{IU} / \mathrm{kg}$ of rhEPO either before or even up to $12 \mathrm{~h}$ after induction of $1 \mathrm{~h} \mathrm{MCAO,} \mathrm{significantly} \mathrm{reduced} \mathrm{the} \mathrm{size} \mathrm{of}$ the brain infarction and extent of inflammation and apoptosis (33) in the ischemic penumbra in rats. In addition, it was demonstrated that rhEpo administration significantly attenuates the neuronal loss in various brain regions after focal transient cerebral ischemia (34). Moreover, administration of the drug exerts decrease in various stages of complex pathophysiological cascades following cerebral ischemia $(12,18)$. Juneman et al. (3) and Im et al. (35) have shown also that reduction of cerebral edema by administration of rhEpo exerts its indirect or secondary neuroprotective effect. Namely, it was shown that administration of rhEpo in rodents caused decrease in swelling of ischemic tissue such as impairment of microcirculation in the critically hypoperfused penumbral area. All these results have shown that rhEpo is drug with neuroprotective potential against cerebral ischemia and that further experiments are needed to confirm these results.

\section{Conclusion}

Our experimental results clearly showed that focal cerebral ischemia induced by MCAO caused significant neuronal damage in the dorsolateral band of the 
entorhinal cortex. Contrary to neuronal loss and neurodegenerative changes in dorsolateral cortex, absence of such results in ventral posteriomedial nucleus of the thalamus is probably due to delayed neuronal response to ischemic changes caused by MCAO in thalamic brain region. Postischemic administration of rhEpo significantly reduced neuronal loss and caused moderate decrease in neurodegeneration. These results clearly indicated neuroprotective potential of rhEpo administered after induction of MCAO.

\section{Acknowledgement}

Grant information: This research was supported by University of Rijeka under project uniri-biomed-18-115 1253 to Jasenka Mršić-Pelčić.

\section{References}

1. Lalkovičová M, Danielisová V. Neuroprotection and antioxidants. Neural Regen Res 2016; 11(6): 865-74.

2. Liu F, McCullough LD. The middle cerebral artery occlusion model of transient focal cerebral ischemia. Methods Mol Biol 2014; 1135: 81-93.

3. Juenemann M, Braun T, Schleicher N, Yeniguen M, Schramm P, Gerriets T et al. Neuroprotective mechanisms of erythropoietin in a rat stroke model. Transl Neurosci. 2020; 11(1): 48-59.

4. Jickling GC, Sharp FR. Improving the translation of animal ischemic stroke studies to humans. Metab Brain Dis 2015; 30(2): 461-7.

5. Simon F, Floros N, Ibing W, Schelzig H, Knapsis A. Neuroprotective potential of erythropoietin after ischemic injury of the central nervous system. Neural Regen Res. 2019; 14(8): 1309-12.

6. Subirós N, García del Barco D, Coro-Antich RM. Erythropoietin: still on the neuroprotection road. Ther Adv Neurol Disord 2012; 5(3): 161-173.

7. Jelkmann W. Physiology and Pharmacology of Erythropoietin. Transfus Med Hemother 2013; 40(5): 302-9.

8. Kaiafa G, Savopoulos C, Kanellos I, Mylonas KS, Tsikalakis G, Tegos T et al. Anemia and stroke: Where do we stand? Acta Neurol Scand 2016; 135(6): $596-602$.

9. Shahab MH, Saifullah Khan S. Erythropoietin Administration for Anemia Due to Chronic Kidney Disease - Subcutaneous OR Intravenous, What Do We Know So Far? Cureus 2020; Sep 10;12(9):e10358. doi: 10.7759/ cureus. 10358. 
10. Al-Qahtani JM, Abdel-Wahab BA, Abd El-Aziz SM. Long-term moderate dose exogenous erythropoetin treatment protects from intermittent hypoxiainduced spatial learning deficits and hippocampal oxidative stress in young rats. Neurochem Res 2014; 39(1): 161-71.

11. Alnaeeli M, Wang L, Piknova B, Rogers H, Li X, Noguchi CT. Erythropoietin in brain development and beyond. Anat Res Int 2012; 1-15. doi: $10.1155 / 2012 / 953264$

12. Wang R, Wu X, Zhao H, Min L, Tao Z, Ji X et al. Effects of erythropoietin combined with tissue plasminogen activator on the rats following cerebral ischemia and reperfusion. Brain Circ 2016; 2(1): 54-60.

13. Minnerup J, Heidrich J, Rogalewski A, Schäbitz WR, Wellmann J. The efficacy of erythropoietin and its analogues in animal stroke models: a metaanalysis. Stroke 2012; 40(9): 3113-20.

14. Pearl RG. Erythropoietin and organ protection: lessons from negative clinical trials. Crit Care 2014; 18(5): 526. doi: 10.1186/s13054-014-0526-9

15. Longa EZ, Weinstein PR, Carlson S, Cummins R. Reversible middle cerebral artery occlusion without craniectomy in rats. Stroke 1989; 20: 84-91.

16. Belayev L, Alonso OF, Busto R, Zhao W, Ginsberg MD. Middle cerebral artery occlusion in the rat by intraluminal suture. Neurological and pathological evaluation of an improved model. Stroke 1996; 27(9): 1616-22.

17. Paxinos G, Watson C. The Rat Brain in Stereotaxic Coordinates. 6th ed. Amsterdam, Netherlands, Academic Press, 2007.

18. Mršić-Pelčić J, Pilipović K, Pelčić G, Vitezić D, Župan G. Decrease in Oxidative Stress Parameters after Post-Ischaemic Recombinant Human Erythropoietin Administration in the Hippocampus of Rats Exposed to Focal Cerebral Ischaemia. Basic Clin Pharmacol Toxicol 2017; 121(6): 453-64.

19. Danielisova V, Gottlieb M, Bonova P, Nemethova M, Burda J. Bradykinin postconditioning ameliorates focal cerebral ischemia in the rat. Neurochem Int 2014; 72: 22-9.

20. Liu F, Schafer DP, McCullough LD. TTC, Fluoro-Jade B and NeuN staining confirm evolving phases of infarction induced by middle cerebral artery occlusion. J Neurosci Methods 2009; 17981: 1-8.

21. Ferrer I, Krupinski J, Goutan E, Marti E, Ambrosio S, Arenas E. Brain-derived neurotrophic factor reduces cortical cell death by ischemia after middle cerebral artery occlusion in the rat. Acta Neuropathol 2001; 101: 229-38. 
22. Duckworth EAM, Butler TL, De Mesquita D, Collier SN, Collier L, Pennypacker KR. Temporary focal ischemia in the mouse: technical aspects and patterns of Fluoro-Jade evident neurodegeneration. Brain Res 2005; 1042(1): 29-36.

23. Onken M, Berger S, Kristian T. Simple model of forebrain ischemia in mouse. J Neurosci Methods 2011; 204(2): 254-61.

24. Delattre C, Bournonville C, Auger F, Lopes R, Delmaire C, Henon H et al. Hippocampal Deformations and Entorhinal Cortex Atrophy as an Anatomical Signature of Long-Term Cognitive Impairment: from the MCAO Rat Model to the Stroke Patient. Transl Stroke Res 2018; 9(3): 294-305.

25. Bordes S, Werner C, Mathkour M, McCormack E, Iwanaga J, Loukas M et al. Arterial Supply of the Thalamus: A Comprehensive Review. World Neurosurg 2020; 137: 310-8.

26. El Amki M, Clavier T, Perzo N, Bernard R, Guichet PO, Castel H.J. Hypothalamic, thalamic, and hippocampal lesions in the mouse MCAO model: Potential involvement of deep cerebral arteries? Neurosci Methods 2015; 254: 80-5.

27. Mitkari B, Kerkelä E, Nystedt J, Korhonen M, Jolkkonen J. Unexpected complication in a rat stroke model: exacerbation of secondary pathology in the thalamus by subacute intraarterial administration of human bone marrowderived mesenchymal stem cells. J Cereb Blood Flow Metab 2015; 35(3): 363-6.

28. Ali Shah F, Li T, Al Kury LT, Zeb A, Khatoon S, Liu G ET AL. Pathological Comparisons of the Hippocampal Changes in the Transient and Permanent Middle Cerebral Artery Occlusion Rat Models. Front Neurol 2019; doi. org/10.3389/fneur.2019.01178

29. Dihné M, Grommes C, Lutzenburg M, Witte OW, Block F. Different Mechanisms of Secondary Neuronal Damage in Thalamic Nuclei After Focal Cerebral Ischemia in Rats. Stroke 2002; 33(12): 3006-11.

30. Carmichael ST. Rodent models of focal stroke: size, mechanism, and purpose. NeuroRx 2005; 2(3): 396-409.

31. Kanazawa M, Takahashi T, Nishizawa M, Shimohata T. Therapeutic Strategies to Attenuate Hemorrhagic Transformation After Tissue Plasminogen Activator Treatment for Acute Ischemic Stroke. J Atheroscler Thromb 2017; 24(3): 240-53. 
Neuroprotective Effect of the Recombinant Human Erythropoietin in the Entorhinal Cortex and Thalamus of Rats Exposed to Focal Cerebral Ischemia

32. Brines M, Cerami A. Erythropoietin and Engineered Innate Repair Activators. Methods Mol Biol 2013; 982: 1-11.

33. Prosvirnina MS, Shmonin AA, Melnikova EV, Vlasov TD. Neuroprotective effects of erythropoietin in focal brain ischemia in rats. Bull Exp Biol Med 2015; 156(5): 642-44.

34. Souvenir R, Doycheva D, Zhang JH, Tang J. Erythropoietin in Stroke Therapy: Friend or Foe. Curr Med Chem 2015; 22(10): 1205-13.

35. Im JH, Yeo IJ, Hwang CJ, Lee KS, Jin Tae Hong JT. PEGylated Erythropoietin Protects against Brain Injury in the MCAO-Induced Stroke Model by Blocking NF$\kappa \mathrm{B}$ Activation. Biomol Ther (Seoul) 2020; 28(2): 152-62. 